\title{
Capítulo VII \\ Del individuo al sujeto crítico, y luego, al sujeto político
}

$\mathrm{D}$ esde que comencé mis estudios en sociología, siempre que leía los textos, me preguntaba a medida que se acercaba el final, ¿con qué propuesta saldrá este autor?, siempre al final de un libro, como lectores, ¡y más como interesados en la política! buscamos que luego de las críticas, aparezcan las propuestas, pues de una manera casi crónica, la crítica política es traducida en "criticadera" por muchos lectores, a mi juicio, por la falta de propuestas de los autores.

¿Qué se puede promover? ¿El cambio del mundo? Que todos los estudiantes y docentes ¿sean los próximos protagonistas de la historia mundial? La verdad, al igual que un reconocido anarquista llamado Buenaventura Durruti, estoy convencido de que si el corazón de alguien es transformado por la utopía, entonces el mundo está cambiando en ese preciso instante. De la misma manera, estoy convencido, como menciona el Papa Francisco, que la constante acumulación de posibilidades para consumir distrae el corazón e impide valorar cada cosa y cada momento (Papa Francisco, 2015) iy es precisamente en la valoración de cada cosa y de cada momento, donde se halla la oportunidad de reflexionar desde el humanismo! 
Finalmente, cuando reflexionas, posibilitas los planos para un sueño, para una utopía que comenzaría a responder ese "¿y qué podemos hacer?" que mencionaba en el capítulo anterior como un interrogante directamente relacionado con lo que significa hablar de política en Colombia.

¿Qué importa si no somos protagonistas de la historia mundial? Al final, la historia mundial está contenida en la historia personal de nuestras vidas, de nuestros familiares y amigos. Mientras se logre constituir y configurar un camino para la reflexión humanista, para el diálogo sobre la política, entonces, puedo advertir que sí soy un convencido de que el mundo se transforma, poco a poco, en el marco del eterno dinamismo, de ideas afines o contrarias que representan la sociología humana, la lógica de ser parte de los que apoyan, de los que contradicen, de los que no se mueven, de los que actúan y viceversa, nadie queda afuera.

En ese marco, el presente capítulo es el último de una serie de consideraciones que he expuesto a lo largo de seis secciones, es la propuesta luego de la reflexión, luego de la andanada de argumentos y contraargumentos que han contenido y desarrollado el objetivo de indagar por las condiciones básicas para hablar de política en Colombia. Ahora, casi al final de este texto, emerge la duda que indica que cuando ya las condiciones básicas para hablar de política están dadas, aquellos sujetos que hablen de política inevitablemente podrían encontrarse con esa pregunta del millón: ¿y qué podemos hacer?

Qué puede surgir luego de que comprendes cuál es el problema de hablar de política en Colombia, de entender que la violencia y la corrupción han marcado el destino de la relación entre los ciudadanos y la política en Colombia, de reconocer que es muy diferente hacer política, hablar de política y escribir pendejadas en las redes sociales, de acercarte a la comprensión de la distinción entre derecha e izquierda en un país donde se confunden la teoría, los propósitos y los actos. Qué pasa cuando descubres que 
los medios masivos de información tienen derecho a decir lo que quieran, pero tú no tienes por qué considerar sus discursos como una realidad objetiva. Qué pasa finalmente cuando te das cuenta de que la democracia tal vez es un fetiche discursivo, que recubre un sistema político que está tan pero tan lejos de ser una democracia o de que te somete con discursos de libertad... Allí, al final de todo esto, a juicio del autor, se ha configurado el plano para una metodología, pues la denuncia solitaria, en una sociedad como la colombiana, podría crear esperpentos de críticos que no son capaces de dar la cara ni por Dios.

En resumen, se trata de comprender dicha metodología como una respuesta que está implícita a lo largo de todo este libro, pero que merece ser racionalizada y expuesta claramente mediante la explicación de dos transformaciones, a saber: la primera, el paso de individuo a sujeto crítico. La segunda, el paso de sujeto crítico a sujeto político. Evidentemente, cada una de las tres categorías implícitas en la propuesta deberán ser definidas, un proceso al que no daré largas, porque las conclusiones, los capítulos finales, deben ser claros y concisos.

¿Quién es el individuo? En el marco de lo que se ha sustentado en los seis capítulos anteriores, el individuo es aquel que vive construyendo los artefactos desprovistos de sentido más absurdos, es aquel que vive sometido a la lógica del consumismo efímero, es un neuroliberal puro que vive en la fantasía del éxito, pero que no logra comprender por qué otros tienen más o por qué nunca podrá ser dueño del país y que desquita su frustración con quien tiene menos que él, en una escala de productos de consumo. $\mathrm{El}$ individuo es el que cree que la realidad es lo que los medios masivos informativos plasman día tras día, noche tras noche, es esa persona que escribe o lee pendejadas en las redes sociales creyendo que allí se habla de política. El individuo es aquel que condujo al hoyo del olvido toda posibilidad colectiva por causa del espectro individualista que ha fomentado en él un sistema mercadófilo. 
El individuo no reflexiona, no se detiene, puesto que detenerse significar pensar, y en el mundo de los individuos, el que piensa, supuestamente pierde; empero, cuando gana, algo adentro le dice que debe ganar más; por lo tanto, sigue siendo perdedor, y así se encierra en un círculo vicioso que no le permite nada más que vivir en la neurosis y obviamente aborreciendo la política en el marco de la programación neuroliberal de la que es preso, pues él y solo él puede alcanzar el éxito que nunca es completo y por lo general se halla por encima de los demás.

Freud eligió el término sublimación para señalar esta extraña transformación que conduce de la represión a la conducta civilizada. Si el volumen de la represión es mayor que la capacidad de sublimación, los individuos se tornan neuróticos y entonces se hace preciso conceder una merma en la represión. (Fromm, 2007, p. 35)

$\mathrm{El}$ individuo se encuentra reprimido, pero trata de negar la realidad mediante el convencimiento de la ficción dicotómica éxito-fracaso. ¿Cómo generar entonces, en términos de Freud, esa capacidad de sublimación para dejar de ser un simple individuo y buscar algo más en el marco de esas facultades humanas que obviamente están reprimidas? En otras palabras ¿cómo dejar de ser un individuo?

"En la proliferación de una ética alternativa a la 'rapacidad del mercado' estriba la clave para trascender la formal remoción de esos obstáculos que, de otro modo, el individuo del neuroliberalismo no rebasaría" (Biagini \& Fernández, 2015, p.118). La batalla definitiva por la primera transformación evidentemente no solo es un proceso de comprensión o acción externa exclusivamente estructural. De hecho, la primera transformación es mucho más comprensiva o minuciosa si se quiere.

Los individuos, al igual que los sujetos críticos, tienen por característica similar, el hecho de que no los conoce nadie, o bueno al menos casi nadie, y en caso de ser aquellos individuos que forman 
parte de los medios masivos televisivos, dicha condición no les exime de las características planteadas anteriormente.

Individuos hay por todas partes. Por lo tanto, la primera transformación no se puede pensar como un fenómeno que inicie de manera estructural. En palabras más claras, si usted es un individuo, no dejará de serlo si no desarrolla una capacidad mínima de sublimación, de cambios en su vida personal, en la vida cotidiana, en la relación con los más próximos, a partir de la voluntad.

¿De dónde emerge la voluntad en estos tiempos donde parece que se pone en duda absolutamente todo? Propongo que emerja de las inquietudes iniciales que se producen en la interacción humana. Probablemente un individuo no dejará de serlo si nunca sale de su casa o cambia los sitios que rutinariamente visita, si no se atreve a cambiar los hábitos, a leer otras realidades más allá de las que se le han presentado siempre como las únicas, invariables y objetivas, es apelar a la facultad humana de la curiosidad. Si el individuo explora, probablemente encuentre algo; si no hay la voluntad, no pasará absolutamente nada.

¿Cómo explicar el hecho de que tanta gente, por ejemplo, se sienta políticamente reprimida por el sistema financiero colombiano, pero sea tan inconsciente a la hora de consumir o votar por los candidatos del sistema financiero? Fromm (2007) ofrece una respuesta analítica muy valiosa:

Cuando una señalada clase se ve amenazada por nuevas tendencias económicas, reacciona frente a tal amenaza tanto psicológica como ideológicamente, y que los cambios psicológicos llevados a cabo por esta reacción contribuyen al ulterior desarrollo de las fuerzas económicas, aun cuando tales fuerzas contradigan los intereses materiales de esa clase. (p. 336)

La reacción ideológica y psicológica que señala Fromm, claramente se puede denominar neuroliberalismo. Dicha reacción ha fomentado que el individuo se niegue o no pueda hablar sobre política, 
porque considera que las cosas son así, que naturalmente son así, o que es mejor, ser como un personaje que en el fondo odia, antes que aceptar el peso de la realidad, comprender la dimensión y darle una oportunidad a la reflexión, y por ende al diálogo sobre la política.

¿Quién es el sujeto crítico? El sujeto crítico es quien, por voluntad inicialmente, logró comprender una o varias facultades humanas que desconocía, hizo frente a la represión en la que vivía, aceptó su lugar en el mundo y se dedicó a comprenderlo. Cabe aclarar que cuando hablo de comprender, no siempre me estoy refiriendo al ejercicio cognoscitivo desarrollado a partir de una plataforma académica; la verdad, como lo he mencionado en alguna ocasión, no existe garantía de que ingresar a una universidad conlleve directamente a la composición de un sujeto crítico; a decir verdad, hemos visto cómo en las universidades en ocasiones no hay críticos sino cretinos, que fomentan constantemente el neuroliberalismo, la exclusión de las humanidades, la virtud del egoísmo y en general todo lo que niegue la probabilidad de la política.

Como el universo de la objetivación y las técnicas se degrada en puro mercado, y el universo de las identidades culturales se encierra en la obsesión comunitaria, el ser particular, el individuo que somos se desgarra, al sentir que su mundo vivido está tan descompuesto como el orden institucional o la representación misma del mundo. (Touraine, 1997, p. 64)

El sujeto crítico es un individuo desgarrado. Se podría afirmar que la primera transformación es sin duda un desgarramiento. El individuo, a medida que va descubriendo cómo funciona el mundo, a medida que va develando las relaciones de poder, la sociología cotidiana, a medida que comprende más allá de la objetivación fallida que siempre le presentaron en forma de discursos mercadotécnicos, cuando comprende que la dicotomía éxito-fracaso no es un marco único de categorización de la vida humana, etc., 
entonces, el individuo sufre una tensión. En primera medida una tensión psíquica, al darse cuenta de que, en términos de Touraine, dos universos siempre lo oprimieron (el del mercado y el de las obsesionadas identidades culturales), siempre lo mantuvieron al margen de poder pensar, de poder detenerse a pensar.

Luego de la tensión, en esa primera transformación, el sujeto crítico se construye a partir de la exaltación de todas aquellas virtudes humanas que son tratadas peyorativamente por el sistema mercadófilo. El sujeto crítico no contiene características o hábitos propios de una élite intelectual que solo disfruta con música clásica o leyendo en silencio todos los días, jen lo absoluto! Eso es un cliché, es un falso modelo de un buen humanista si dichas características son atributos "necesarios" y excluyentes.

El sujeto crítico vive la vida, pero no la vivirá jamás de forma engañosa, es un sujeto que conoce, que se tensiona por sus acciones, pero también se gratifica cuando logra comprender que su vida puede ser verdaderamente libre si explora todas las condiciones humanas que tiene a su disposición y fueron negadas desde siempre por una institución, una ideología o un fetiche. El sujeto crítico no es exclusiva y excluyentemente el que más lee, ese tipo de clichés, pronto constituyen penosas falacias; como decía, una pancarta anónima colocada en las alamedas de la Universidad de los Andes en Bogotá a propósito de los doctores: "tenemos 406 profesores con doctorado, no sabemos si son buenos profesores, pero tienen doctorado" anónimo. 
Fotografia 3. Pancarta en la Universidad de los Andes, 2014

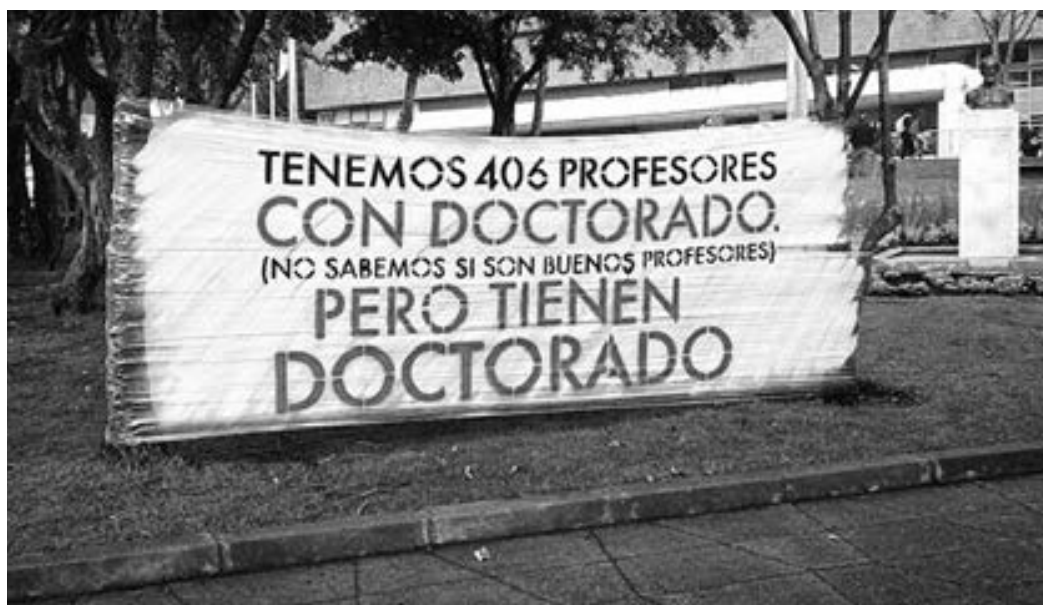

Fuente: tomada de http://akalpadev.blogspot.com.co/2014/11/el-conocimientocientifico-y-la.html

El sujeto crítico, entonces, es absolutamente libre. Libre de la libertad como fetiche, libre del mercado como fetiche, libre de la democracia como fetiche, libre porque exalta el elemento más valioso que posee, un elemento cargado de facultades que solo puede extraer mediante la reflexión personal y la reflexión con los demás, un elemento que no puede descubrir solo. No. No se puede porque sin libertad colectiva no existe sino un espectro de libertad individual.

Necesitamos a los demás, ese elemento es: ser humanos. Y ser consciente de ese elemento es también una condición básica para hablar de política en Colombia. ¿Quién es finalmente el sujeto político? El sujeto político emerge de la segunda transformación. ¿ $\mathrm{Ha}$ sentido en ocasiones molestia cuando intenta explicar una injusticia y las personas le responden con argumentos incoherentes, indiferencia o burla? ¿Recuerda aquella vez que pensó o gritó luego de conocer una noticia perversa, "en Colombia no pasa nada”? ¿Ha sido consciente que en ocasiones perdió la tranquilidad y entró en 
desespero, por querer hacer algo correcto en la vida cotidiana y fue tratado de "sapo", amedrentado con groserías o golpeado injustamente? Bien, la segunda transformación la caracterizan dos necesidades que contiene implícitas un sujeto crítico.

La primera, conseguir reducir la angustia que genera el desgarramiento. Dicha angustia, se reduce a medida que el sujeto crítico comprende que su voluntad debe ser alimentada, nutrida y sobre todo retroalimentada. ¿Por quién? Allí nace la necesidad de asociación, el escenario para la política. Touraine (1997) expone teóricamente, la caracterización que deseo realizar de la segunda transformación, la expone como un doble movimiento.

El doble movimiento solo puede ser efectuado por un sujeto personal cuyas virtudes no son las que exige la ética de la discusión. La primera es el valor solitario (que denuncia a los poderes), y la segunda, la fuerza de acción colectiva, que permite que el sujeto sobreviva. (p. 66)

Valor solitario y acción colectiva, son las características de la segunda transformación; el sujeto político no es el que se empeña en convertirse en un personaje histórico, sino el que se empeña en transformar la realidad social mediante la política. La política de los sujetos. Los que no son mediados por fuerza externa, sino que, mediante el diálogo sobre política, confluyen, concluyen y conllevan a la acción. ¿Qué tipo de acción? ¿Es posible determinar la acción política mediante una abstracción teórica? Yo pienso que no. No se pueden dar "recetas" del quehacer si los sujetos políticos no se han sentado a hablar sobre un contexto y una situación particular.

El sujeto político está ajustado no por la teoría, sino por el contexto y las probabilidades medibles y concretas de su acción. Una acción que no puede ser solitaria, pues es el resultado del diálogo sobre la política, es política en el sentido más puro de la palabra.

Como conclusión, el paso por las dos transformaciones no constituye obligaciones inamovibles propias de la forma típica como 
recibimos las teorías. Considero mucho más acertado convenir en que, sin develar y apropiarse de las condiciones básicas para hablar de política en Colombia, simplemente, las personas preocupadas por no saber qué hacer o cómo hacer seguirán siendo lobos solitarios, que, en el peor de los casos, establecerán discursos en los que indiquen que se "cansaron", que "no hay salida" solo como resultado de que no pudieron efectuar la segunda transformación.

Hablar de política, sin lugar a dudas, constituye en Colombia el escenario de las probabilidades, es decir, si se desarrolla seriamente un diálogo sobre política se descubrirá que existen mil cosas por hacer, se descubrirá que la política en lo absoluto no es "una mierda", y que promover las condiciones básicas para hablar de la misma, podría constituirse como aquella primera transformación necesaria para forjar sujetos críticos.

Ahora bien, la segunda transformación, el ser sujetos políticos, dependerá del valor solitario, de la voluntad, de la capacidad de asociación con el otro, por el otro, y nada más que el otro, para que cuando juntos comprendan el acto que propenderá una real liberación, gocen a plenitud su individualidad, pudiendo pronosticar una idea más lejana que una hipótesis y más cercana que una utopía, que dicha individualidad será profunda y sincera, porque será producto del quehacer colectivo... un camino que jamás será el último, pero que podríamos tomar, a ver qué sucede. El debate queda abierto... 


\section{Epílogo}

Condiciones básicas para hablar de política en Colombia fue, ante todo,
un conjunto de pautas o bien, puntos focales con los cuales quise llamar la atención, sobre todo de aquellos y aquellas estudiantes que durante mi ejercicio docente han sentido gran parte o la totalidad de las preocupaciones aquí planteadas. Como es justo y lógico, la crítica puede emerger, no obstante, la intención que nunca fue subliminal y que por propositiva no sería justo catalogarla de contener intenciones "mesiánicas", creo que por su lenguaje se apartó de un tedioso manual sobre política, creo que cumplió su objetivo: llegar a los estudiantes.

Los estudiantes universitarios son las personas que pueden desarrollar, debatir, destruir o reconfigurar de mejor manera y con más repercusión, todas las ideas aquí presentadas. Por otra parte, la gente común, la gente que forma y no forma parte de la academia, también puede encontrar en medio de todo lo que fue este texto, una serie de vórtices sobre los cuales al menos varias de las muchas preguntas planteadas a lo largo del libro, pueden o no servir para orientar formas de pensar problemas políticos coyunturales.

Por último, siempre seré un convencido de que la comunicación con el autor de un texto es más que necesaria a la hora de poder establecer diálogos constructivos sobre ideas planteadas. Por lo tanto, dejo a merced del lector o lectora, la decisión de comunicarse conmigo si así lo requiere. Con gusto y de seguro, hablaremos de política en Colombia. 



\section{Bibliografía}

Aguilera, M. (1985). Los comuneros guerra social y lucha anticolonial. Bogotá, Colombia: Universidad Nacional.

Andrade, E. (1990). Introducción a la ciencia política. México D.F., México: Harla.

Arendt, H. (2005). Sobre la violencia. Madrid, España: Alianza.

Atehortúa, A. (2010, abril). El golpe de Rojas y el poder de los militares. $F_{0}$ lios, (31), 33-48. Recuperado de http://www.scielo.org.co/pdf/folios/ n31/n31a03.pdf

Banco de la República. (s. f.). La Bagatela. Recuperado de http://www.banrepcultural.org/blaavirtual/historia/la-bagatela/indice.htm

Beltrán, L. R. (2005). La comunicación para el desarrollo en Latinoamérica: Un recuento de medio siglo. Buenos Aires, Argentina: Universidad de Buenos Aires.

Biagini, H., \& Fernández, D. (2015). El neuroliberalismo y su ética gladiatoria. En Biagini, H., y Peychaux, D. (Comp), Democracia, neoliberalismo y pensamiento político alternativo (pp. 115-122). Buenos Aires, Argentina: Universidad Nacional de Lanús.

Bobbio, N. (1995). Derecha e Izquierda. Razones y significados de una distinción politica. Roma, Italia: Taurus.

Bourdieu, P. (Enero 1972). La opinión pública no existe. Conferencia llevada a cabo en Noroit (Arras). Recuperado de http://sociologiac.net/biblio/ Bourdieu_OPE.pdf

Cascales, A., Real, J. J., \& Marcos, B. (2011). Redes sociales en internet. Revista electrónica de tecnología educativa, (38), 1-18. Recuperado de http:// www.edutec.es/revista/index.php/edutec-e/article/view/381/118 
Central Unitaria de Trabajadores de Colombia (CUT). (2013). Masacre de las Bananeras diciembre 6 de 1928. Recuperado de http:/ / comunicandes. org/pdf/2013/MASACRE\%20DE\%20LAS\%20BANANERAS.pdf

Cortés, H. (2011). Los comuneros. ¿Una revuelta anti-colonial? (tesis de pregrado). Recuperado de http://www.bdigital.unal.edu.co/7171/1/heidicortesacosta.2012.pdf

Contravía, M. (Productor). (2007). Confesiones de un exparamilitar [Youtube]. Recuperado de https://www.youtube.com/watch?v=TOFsMqEhDeQ

Departamento Administrativo Nacional de Estadística (DANE). (2015). Tercer Censo Nacional Agropecuario. Recuperado de http://wp.presidencia. gov.co/Noticias/2015/Agosto/Paginas/20150811_06-DANE-entrega-avance-de-resultados-del-tercer-Censo-Nacional-Agropecuario.aspx

ElTiempo.com. (11 de marzo de 2015). "La ética no tiene nada que ver con el derecho" dice De la Espriella. El Tiempo. Recuperado de http:/ /www. eltiempo.com/politica/justicia/la-etica-no-tiene-nada-que-ver-con-elderecho-abelardo-de-la-espriella/15378415

Friedman, M. (1979). Libertad de elegir. Nueva York, Estados Unidos: Orbis, S. A.

Fromm. E. (2007). El miedo a la libertad. Buenos Aires, Argentina: Paidós.

Gadamer, H. (1998). El giro hermenéutico. Madrid, España: Ediciones Cátedra.

Gaitán, J. E. (1946). Discurso de la candidatura liberal para las elecciones de mayo de 1946. Teatro Municipal de Bogotá. Recuperado de https://es.wikisource.org/wiki/Discurso_en_el_Teatro_Municipal_de_1946

Goffman, E. (1959). La presentación de la persona en la vida cotidiana. Buenos Aires, Argentina: Amorrortu.

Islas-Carmona, J. O. (2008). El prosumidor. El actor comunicativo de la sociedad de la ubicuidad. Palabra-Clave, 11(1), 29-39. Recuperado de http:/ / www.redalyc.org/articulo.oa?id=64911103

Kalmanovitz, S. (1985). El desarrollo histórico del campo colombiano. Recuperado de http://www.banrepcultural.org/blaavirtual/historia/colhoy/colo9.htm

Klein, Y. (Productor). (16 de noviembre de 2012). No he dicho ninguna mentira, no he tenido ninguna contradicción (...) di detalles adicionales y más precisos para que la verdad tenga más sustentos [Audio podcast]. Recuperado de http://www.wradio.com.co/escucha/archivo_de_audio/ 
klein-no-he-dicho-ninguna-mentira-no-he-tenido-ninguna-contradiccion--di-detalles-adicionales-y-mas-precisos-para-que-la-verdad-tengamas-sustentos/20121116/oir/1796524.aspx

König, H. (1994). En el camino hacia la nación, nacionalismo en proceso de formación del Estado y de la nación de la Nueva Granada, 1750-1856. Bogotá, D. C., Colombia: Banco de la República.

Lechner, N. (1996). La política ya no es lo que fue. Nueva sociedad, (144), 70-86. Recuperado de http://nuso.org/media/articles/downloads/2518_1.pdf

Lenin, V. (1917). El programa militar de la revolución proletaria. Recuperado de https://www.marxists.org/espanol/lenin/obras/1910s/1916mil.htm

Lenk, K. (2000). El concepto de ideología, comentario crítico y selección sistemática de textos. Buenos Aires, Argentina: Amorrortu editores.

Liévano, I. (2002). Los grandes conflictos sociales y económicos de nuestra historia. Bogotá, Colombia: Intermedio.

Liévano, I. (2013). Bolivar. Bogotá, Colombia: Grijalbo.

Lourdes, V. (2006). Cuando la Guerra Fría llegó a América Latina ... La Política Exterior Norteamericana hacia Latinoamérica durante las presidencias de Eisenhower y Kennedy (1953-1963). Centro Argentino de Estudios Internacionales. Recuperado de http://www.caei.com.ar/working-paper/cuando-la-guerra-fr $\% \mathrm{C} 3 \% \mathrm{AD}-\mathrm{lleg} \% \mathrm{C} 3 \% \mathrm{~B} 3-\mathrm{am} \% \mathrm{C} 3 \% \mathrm{~A} 9$ rica-latina-la-pol $\% \mathrm{C} 3 \% \mathrm{~A}$ Dtica-exterior-norteamericana-hacia

Marx, C. (1989). Contribución a la crítica de la economía politica. Moscú, Rusia: Progreso.

Murga, J. (2015). 7 mecanismos de defensa inconsciente. Recuperado de http:// www.recursosdeautoayuda.com/7-mecanismos-de-defensa/

Nariño, A. (28 de julio de 1811). Continuación del Gobierno de los Estados Unidos. La Bagatela. Recuperado de http://www.banrepcultural. org/sites/default/files/lablaa/historia/la-bagatela/la-bagatela2.pdf

Ocampo, J. (2009). La independencia de Colombia. Bogotá, Colombia: Bicentenarios de América Latina.

Papa Francisco. (24 de mayo de 2015). Carta encíclica. Laudato si'. Recuperado de: http://w2.vatican.va/content/francesco/es/encyclicals/documents/papa-francesco_20150524_enciclica-laudato-si.html 
Proudhon, P. (2005) ¿Qué es la propiedad? Investigaciones sobre el principio del derecho y del gobierno. Buenos Aires, Argentina: Libros de Anarres.

Quintana, J. (2007). En los albores de la 'Ideología' en España. Historia de la psicología, 28(2/3), 205-211. Recuperado de https://dialnet.unirioja. es/servlet/articulo?codigo $=2383454$

Quintero, L. (2008). Los "pájaros" del Valle del Cauca. Estudios de derecho. 243-258.

Rivas, L., \& Rosado, J. (2007). María Cano 1887- 2007. Una voz de mujer les grita. Medellín, Colombia: Escuela Nacional Sindical.

Ricoeur, P. (2002). Del texto a la acción. Ensayo de hermenéutica II. México, D.F., México: Fondo de Cultura Económica.

Rose-Ackermaan, S. (2001). La corrupción y los gobiernos: causas, consecuencias y reforma. Madrid, España: Siglo XXI.

Sacks, O. (1987). El hombre que confundió a su mujer con un sombrero. Barcelona, España: Anagrama.

Semana.com (14 de mayo de 2016). "Hacer invivible la república" dice Laureano Gómez. Semana. Recuperado de http://www.semana.com/ opinion/articulo/antonio-caballero-resistencia-civil-de-alvaro-uribe-fomenta-guerra-civil/473487

Thoreau, H. (1980). Del deber de la desobediencia civil. Buenos Aires, Argentina: Editorial Pi.

Touraine, A. (1997). ¿Podremos vivir juntos? Iguales y diferentes. México, D.F. México: Fondo de Gultura Económica.

Uribe, R. (1904). Socialismo de Estado. Conferencia dictada en el Teatro Municipal de Bogotá. Recuperado de http://cruzadasur.blogspot.com. co/2012/01/socialismo-de-estado.html

Uribe, R. (1910). Los problemas nacionales. Bogotá, Colombia: imprenta eléctrica.

Verdadabierta.com. (2008). Masacres. Recuperado de http://www.verdadabierta.com/masacres-seccion/596-masacres-1997-2001-

Weber, M. (1919). El político y el científico. Recuperado de http://www.hacer. $\mathrm{org} / \mathrm{pdf} /$ WEBER.pdf 
Žižek, S. (2000). Mirando el sesgo. Una introducción a faques Lacan a través de la cultura popular. Buenos Aires, Argentina: Paidós.

Žižek, S. (2001). Slavoj Žižek en español. Rio de Janeiro, Brasil: Record. 

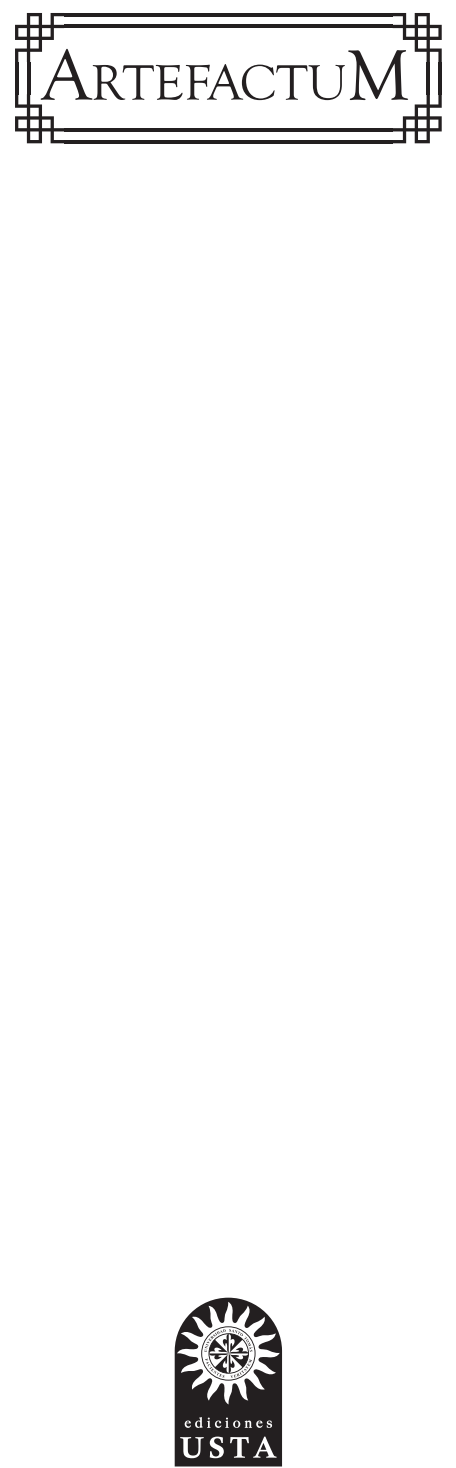

Esta obra se editó en Ediciones USTA,

Departamento Editorial de la Universidad Santo Tomás. Se usó papel propalcote de 300 gramos para la carátula y papel bond beige de 75 gramos para páginas internas.

Tipografia de la familia Baskerville Style BT 2017. 
${ }^{1}$ presente texto es una reflexión que surge a partir del diálogo directo con los estudiantes, con sus preocupaciones, sus inquietudes, sus dudas, su desconocimiento, sus certezas, utopías, distopías, desesperanzas, desconfianzas y anhelos frente a los escenarios políticos que reconocen. Por lo anteriormente mencionado, el presente texto significa, nada más que el propósito de escribir a ese grupo esencial de la universidad: los estudiantes, sin distinción de género o cultura.

El lector hallará aquí referencias teóricas claras, referencias bibliográficas explícitas y propuestas lógicas que darán cuenta del tratamiento conceptual a las problemáticas que se plantearán capítulo a capítulo. Empero, y como sana advertencia, si el lector espera encontrarse un tratado sobre política, se llevará una gran decepción, ya que atendiendo a un clamor de base, fáctico y esencial, el presente libro, sociológicamente se puede anunciar ni más ni menos como un texto cuyas características más cercanas son la formulación de preguntas, el metalenguaje, la exclamación, el salto atemporal histórico, al mejor estilo de las películas que van pasando las partes del final desde el comienzo y luego se devuelven obligando a los espectadores a tejer autónomamente formulaciones lógicas de posibles desenlaces para estructurar una comprensión general. 